\title{
Incidence, prevalence and mortality of type 2 diabetes requiring glucose-lowering treatment, and associated risks of cardiovascular complications: a nationwide study in Sweden, 2006-2013
}

\author{
Anna Norhammar ${ }^{1,2}$ - Johan Bodegård ${ }^{3}$ - Thomas Nyström ${ }^{4}$ - Marcus Thuresson ${ }^{5}$. \\ Jan W. Eriksson ${ }^{6}$ - David Nathanson ${ }^{4}$
}

Received: 5 November 2015 / Accepted: 22 March 2016 /Published online: 18 May 2016

(C) Springer-Verlag Berlin Heidelberg 2016

\begin{abstract}
Aims/hypothesis The global diabetes epidemic affects countries differently. We aimed to describe trends in the incidence and prevalence of type 2 diabetes mellitus requiring glucoselowering treatment, together with associated life expectancy and risks of significant clinical complications.

Methods Data on patients with type 2 diabetes who filled a prescription for any glucose-lowering drug (GLD) during the period 2006-2013 were extracted from the Swedish Prescribed Drug Register, Cause of Death Register and Swedish National Patient Register.

Results In 2013, the prevalence of GLD-treated type 2 diabetes was $4.4 \%(n=352,436)$ and the incidence was 399 per 100,000 population $(n=30,620)$. During 2006-2013, the prevalence increased by $61 \%$ while the incidence remained relatively stable;
\end{abstract}

Electronic supplementary material The online version of this article (doi:10.1007/s00125-016-3971-y) contains peer-reviewed but unedited supplementary material, which is available to authorised users.

Anna Norhammar

anna.norhammar@ki.se

1 Cardiology Unit, Department of Medicine, Solna, Karolinska Institutet, Karolinska University Hospital, 171

76 Stockholm, Sweden

2 Capio St Görans Hospital, Stockholm, Sweden

3 Astra Zeneca Nordic-Baltic, Södertälje, Sweden

4 Department of Clinical Science and Education, Division of Internal Medicine, Unit for Diabetes Research, Karolinska Institutet, Södersjukhuset, Stockholm, Sweden

5 Statisticon AB, Uppsala, Sweden

6 Department of Medical Sciences, Clinical Diabetes and Metabolism, Uppsala University, Uppsala, Sweden the prevalence of cardiovascular disease (CVD, 34\% in 2013) and microvascular disease (16\% in 2013) was also stable. Insulin use increased by $29 \%$ while sulfonylurea use declined by $55 \%$. Compared with the general population, patients with type 2 diabetes had increased risk of myocardial infarction, stroke and allcause mortality, with age-standardised risks of $\sim 1.7-, 1.5$ - and 1.3-fold, respectively. These risks declined over time. Life-years lost due to diabetes was most pronounced at younger ages and improved in women over time from 2006 to 2013.

Conclusions/interpretation The prevalence of type 2 diabetes requiring GLD treatment in Sweden increased substantially in recent years, while the incidence remained stable. Use of sulfonylurea declined while insulin use increased. The high prevalence of diabetes-related comorbidities, increased risk of complications and life-years lost highlights the need for optimised and new preventive strategies in patients with type 2 diabetes.

Keywords Cardiovascular disease · Glucose-lowering therapy $\cdot$ Incidence $\cdot$ Life expectancy $\cdot$ Mortality $\cdot$ Prevalence . Type 2 diabetes

$\begin{array}{ll}\text { Abbreviations } \\ \text { ATC } & \text { Anatomical therapeutic chemical } \\ \text { CVD } & \text { Cardiovascular disease } \\ \text { GLD } & \text { Glucose-lowering drug } \\ \text { NBHW } & \text { National Board of Health and Welfare } \\ \text { NDR } & \text { National Diabetes Register } \\ \text { PDR } & \text { Prescribed drug register }\end{array}$

\section{Introduction}

The global diabetes epidemic affects both western and developing societies; 387 million people (8-9\%) worldwide were 
living with diabetes in 2014 [1, 2]. This scenario is paralleled by the obesity epidemic [1]. Both diabetes and obesity reduce life expectancy, mainly owing to complications from diabetes and cardiovascular disease (CVD) [2,3]. In the most recent report from the WHO on non-communicable disease, diabetes was highlighted in two of the nine major global targets for prevention and control [2]. The aim is to halt the global rise in diabetes and obesity and to achieve a $25 \%$ reduction in the overall mortality from CVD, cancer, respiratory disease and diabetes by the year 2025 [2].

A large proportion of those with diabetes, however, are undiagnosed, and therefore do not have the opportunity to benefit from available effective preventive measures. Life expectancy of people with diabetes has increased in western societies; however, an earlier onset of diabetes and a decline in diabetes-related mortality leads to a prolonged time at risk of complications of the disease as well as to an increased diabetes prevalence [4].

Recent figures from Sweden show that despite a rise in prevalence of obesity and of being overweight [5], both the prevalence and incidence of diabetes are unchanged $[6,7]$ or only slightly increasing [8]. Considering the ageing population in Sweden, and that levels of physical inactivity are unchanged and obesity is increasing, this finding is unexpected [4]. Moreover, increased immigration from countries with a high prevalence of diabetes might be expected to increase the overall prevalence in Sweden [9].

It is important to estimate accurately the burden and related complications of type 2 diabetes mellitus, which is the most common type of diabetes. Any underestimation could impede health authorities' future economic planning and provision of preventive care. The main aim of this nationwide study was to provide updated information from the entire population in Sweden on the current incidence and prevalence of type 2 diabetes treated with a glucose-lowering drug (GLD), and the trends in incidence and prevalence in recent years. A further aim was to describe trends in GLD use and investigate CVD risk, mortality and life expectancy in patients with type 2 diabetes compared with the general population.

\section{Methods}

Data sources This was an observational registry study utilising three national Swedish registries: the Prescribed Drug Register (PDR) 1 July 2005 to 31 December 2013, covering all drug prescriptions filled using Anatomical Therapeutic Chemical (ATC) codes; the Cause of Death Register 1961-2013; and the National Patient Register covering all hospital admissions and discharge diagnoses in 1987-2013, discharge diagnoses and open patient clinic visits in 2001-2013. All three registers are held by the Swedish National Board of Health and Welfare (NBHW).
Study population All patients with any prescribed GLD (ATC code A10) found in the PDR from 1 July 2005 to 31 December 2013 were included in the dataset. The index date was defined as the first registered occasion of a filled prescription of a GLD. Patients with a diagnosis of gestational diabetes (ICD-10 code: O24.4; www.who.int/classifications/icd/en) within 1 year of the index date and patients with type 1 diabetes were excluded. Patients with type 1 diabetes were defined as those with a type 1 diabetes diagnosis (ICD-10 code E10) and treated with insulin during their first year of GLD treatment, or aged under 30 years at the start of insulin treatment, or aged under 15 years at the start of any diabetes medication. Patients were observed until death or the end of the year 2013. Individual patient-level data from the national registers were linked using personal identification numbers. Data linkage was performed by the NBHW and the linked database was managed by Statisticon AB, Uppsala, Sweden. The study protocol was approved by the Stockholm regional ethics committee (registration number 2013/2206-31).

Definitions Comorbidities and treatments, defined by ICD diagnoses and ATC codes, were identified for the type 2 diabetes population at each calendar year based on main discharge diagnosis for CVD and based on both main and secondary diagnoses for other diseases (see Electronic Supplementary Material [ESM] Table 1). Life expectancy was calculated for the mean age of the type 2 diabetes cohort, at 67 years.

Statistical analysis The incidence and prevalence of GLDtreated type 2 diabetes was calculated for each year from 2006 to 2013, using the total Swedish population aged over 15 years, at each year, as the reference. Annual prevalence and mortality rates were age stratified into two groups, $\geq 70$ and $<70$ years to show age-related contributions. For incidences, the denominator was the type 2 diabetes-free population (the total population at the end of the year of interest minus the population with type 2 diabetes at the end of the year before the year of interest). Patients with newly initiated GLD treatment were defined when first dispense of GLD was registered after being treatment naive for one year.

Calendar year patient data are presented as the mean and SD for continuous variables, and numbers and percentages for categorical variables. Age-adjusted annual risks of hospitalisation with a main diagnosis of myocardial infarction (ICD-10 I21), stroke (I63), heart failure (I50) or atrial fibrillation (I48), and total mortality for the type 2 diabetes population were compared with those for the Swedish general population using indirect standardisation. Interaction tests were performed to assess risk ratio differences between men and women. Data for the Swedish population were collected from Statistics Sweden (population size) and the NBHW (number of patients with a hospitalisation). Life expectancy was estimated by the Chiang's method for those with and without diabetes [10]. All analyses 
were conducted using $\mathrm{R}$ statistical software ( $\mathrm{R}$ version 3.1.1) [11]. Life-years lost were calculated by subtracting life expectancy in patients with type 2 diabetes from that in the general population.

\section{Results}

Incidence and prevalence of type 2 diabetes During the observation period, the prevalence of type 2 diabetes requiring treatment with GLDs in Sweden increased by $61 \%$, from $2.7 \%$ $(n=207,303)$ in 2006 to $4.4 \%(n=352,436)$ in 2013 (Table 1$)$. Age-stratified analyses of prevalence show that the age group $<70$ years contributes to a larger extent to the annual prevalence due to its bigger size compared with the group $\geq 70$ years, see ESM Fig. 1. However, the proportion of the total type 2 diabetes population between patients $<70$ and $\geq 70$ years ( 55 and $45 \%$, respectively) seems to be stable over the years indicating a stable and proportional contribution from both groups (ESM Fig. 1). ESM Fig. 2 shows that the age group $\geq 70$ contributes a larger proportion of mortality cases $(\sim 3.0 \%$ in $2013)$ compared with the younger age group ( $0.6 \%$ in 2013$)$ relative to the whole type 2 diabetes population. The change in mortality from 2006 to 2013 is only small $(2.8 \%, n=4,006$ deaths) when compared with the total change in prevalence (145,133 increased number of type 2 diabetes patients). The incidence was relatively stable over these years, with a mean of 420 new cases per 100,000 inhabitants annually (Table 1). The estimated incidence and prevalence did not change substantially in sensitivity analyses in which incident cases were defined as requiring a second or a third prescription of a GLD (data not shown).

Type 2 diabetes population Overall 253,689 patients had a prescription of a GLD filled for the first time during the observation period. Mean age of patients in 2013 was 67 years and $44 \%$ were female (Table 2). Annual prevalence of history of CVD increased slightly to $34.2 \%$, with no changes in prevalence of myocardial infarction $(10.1 \%)$ or stroke $(10.3 \%)$. Prevalence of heart failure increased slightly (to $5.8 \%$ ), with a more pronounced increase in prevalence of atrial fibrillation $(+28.3 \%)$ to $8.6 \%$ (Table 2). The annual prevalence of a history of microvascular disease was stable at $\sim 26 \%$ and severe hypoglycaemia was uncommon. Although relatively rare, kidney disease doubled in prevalence from $2.5 \%$ to $4.9 \%$. The prevalence of cancer increased significantly to $18.7 \%$.

During the observation period the mean age of patients newly initiated on GLD treatment dropped slightly but remained similar overall (ESM Table 2); the number of patients burdened with CVD and microvascular disease also dropped slightly.

Drug treatment patterns The overall use of CVD-preventive drugs was stable over the years and $\sim 84 \%$ of patients had one or more prescriptions filled for antihypertensive treatment, statins or low-dose aspirin (Table 2). Statin use increased $(+20.8 \%)$ while the use of low-dose aspirin decreased ($17.5 \%$ ). In 2013, the most frequently used GLDs were metformin, insulin and sulfonylurea (Table 3, Fig. 1). During the observation years, the use of insulin secretagogues was reduced by more than half; in contrast, the use of insulin increased substantially. Despite a steep increase in the later years, use of incretin-based treatment remained low in $2013(9 \%)$. The use of blood glucose test strips decreased markedly (Table 3).

\section{CVD incidence, all-cause mortality and life expectancy} From 2006 to 2013, the age-standardised event rates in the general Swedish population and in the type 2 diabetes population for myocardial infarction, ischaemic stroke and all-cause death showed an overall decrease, while the rate for atrial fibrillation showed an overall numerical increase (Table 4). Compared with

Table 1 Crude incidence, prevalence and population at risk of type 2 diabetes requiring treatment with GLD

\begin{tabular}{|c|c|c|c|c|c|c|c|c|c|c|}
\hline & 2006 & 2007 & 2008 & 2009 & 2010 & 2011 & 2012 & 2013 & $\begin{array}{l}\text { Change (\%) } \\
\text { from } 2006 \\
\text { to } 2013\end{array}$ & $\begin{array}{l}p \text { for } \\
\text { trend }\end{array}$ \\
\hline Swedish population, $n$ & $7,574,846$ & $7,650,473$ & $7,723,931$ & $7,800,212$ & $7,861,268$ & $7,909,176$ & $7,953,511$ & $8,006,339$ & 5.7 & $<0.001$ \\
\hline Type 2 diabetes population ${ }^{\mathrm{a}}, n$ & 207,303 & 227,837 & 249,495 & 270,284 & 293,013 & 313,599 & 334,360 & 352,436 & 70.0 & $<0.001$ \\
\hline Prevalence $(\%)$ & 2.7 & 3.0 & 3.2 & 3.5 & 3.7 & 4.0 & 4.2 & 4.4 & 60.8 & $<0.001$ \\
\hline Swedish population ${ }^{\mathrm{b}}, n$ & $7,388,283$ & $7,443,170$ & $7,496,094$ & $7,550,717$ & $7,590,984$ & $7,616,163$ & $7,639,912$ & $7,671,979$ & 3.8 & $<0.001$ \\
\hline $\begin{array}{l}\text { Incident type } 2 \text { diabetes } \\
\quad \text { population }^{\mathrm{c}}, n\end{array}$ & 34,020 & 29,261 & 31,226 & 30,966 & 33,332 & 31,853 & 32,411 & 30,620 & -10.0 & 0.865 \\
\hline Incidence per $100,000, n$ & 460 & 393 & 417 & 410 & 439 & 418 & 424 & 399 & -13.3 & 0.436 \\
\hline $\begin{array}{l}\text { Deaths in type } 2 \text { diabetes } \\
\text { population }{ }^{\mathrm{d}},(n, \%)\end{array}$ & $\begin{array}{l}8,727 \\
(4.2)\end{array}$ & $\begin{array}{r}9,568 \\
(4.2)\end{array}$ & $\begin{array}{r}10,177 \\
(4.1)\end{array}$ & $\begin{array}{r}10,603 \\
(3.9)\end{array}$ & $\begin{array}{r}11,267 \\
(3.8)\end{array}$ & $\begin{array}{r}11,650 \\
(3.7)\end{array}$ & $\begin{array}{r}12,544 \\
(3.8)\end{array}$ & $\begin{array}{r}12,733 \\
(3.6)\end{array}$ & -14.2 & $<0.001$ \\
\hline
\end{tabular}

Changes are calculated for original values before approximation to one decimal

${ }^{\text {a }}$ Diabetes population $=$ patients with type 2 diabetes treated with GLDs

${ }^{\mathrm{b}}$ Swedish population $=$ all Swedish inhabitants older than 15 years, with or without type 2 diabetes

${ }^{\mathrm{c}}$ For incidence, the denominator was the type 2 diabetes-free population

${ }^{\mathrm{d}}$ Total number of deaths in the type 2 diabetes population. Percentage in brackets is number of deaths divided by the total type 2 diabetes population 
Table 2 Annual baseline prevalent characteristics of patients with type 2 diabetes requiring treatment with GLDs

\begin{tabular}{|c|c|c|c|c|c|c|c|c|c|c|}
\hline & 2006 & 2007 & 2008 & 2009 & 2010 & 2011 & 2012 & 2013 & $\begin{array}{l}\text { Change from } \\
2006 \text { to } 2013\end{array}$ & $\begin{array}{l}p \text { for } \\
\text { trend }\end{array}$ \\
\hline Number of patients & 207,303 & 227,837 & 249,495 & 270,284 & 293,013 & 313,599 & 334,360 & 352,436 & $70.0 \%$ & $<0.001$ \\
\hline Age (years) & 67.7 & 67.5 & 67.3 & 67.2 & 67.1 & 67.0 & 67.0 & 67.1 & -0.9 years & 0.002 \\
\hline Female sex $(\%)$ & 45.1 & 45.0 & 44.8 & 44.6 & 44.4 & 44.2 & 44.0 & 43.9 & $-2.7 \%$ & $<0.001$ \\
\hline CVD $(\%)$ & 32.6 & 33.0 & 33.2 & 33.4 & 33.6 & 33.8 & 34.1 & 34.2 & $5.0 \%$ & $<0.001$ \\
\hline Myocardial infarction (\%) & 10.1 & 10.2 & 10.3 & 10.2 & 10.2 & 10.1 & 10.1 & 10.1 & $-0.3 \%$ & 0.239 \\
\hline STEMI (\%) & 4.8 & 4.8 & 4.7 & 4.6 & 4.5 & 4.4 & 4.4 & 4.3 & $-11.2 \%$ & $<0.001$ \\
\hline NSTEMI (\%) & 6.5 & 6.7 & 6.9 & 6.9 & 7.0 & 7.1 & 7.2 & 7.1 & $9.5 \%$ & $<0.001$ \\
\hline Unstable angina $(\%)$ & 4.7 & 4.8 & 4.9 & 4.9 & 4.9 & 4.8 & 4.8 & 4.7 & $-0.1 \%$ & 0.532 \\
\hline Angina pectoris (\%) & 10.0 & 10.0 & 10.1 & 10.0 & 10.0 & 10.0 & 10.0 & 9.9 & $-0.6 \%$ & 0.237 \\
\hline Stroke $(\%)$ & 10.3 & 10.3 & 10.3 & 10.2 & 10.2 & 10.2 & 10.3 & 10.3 & $0.2 \%$ & 0.995 \\
\hline Haemorrhagic (\%) & 0.5 & 0.6 & 0.6 & 0.6 & 0.7 & 0.7 & 0.7 & 0.7 & $37.7 \%$ & $<0.001$ \\
\hline Embolic (\%) & 8.0 & 7.9 & 7.8 & 7.8 & 7.7 & 7.5 & 7.5 & 7.4 & $-7.5 \%$ & $<0.001$ \\
\hline Heart failure (\%) & 5.5 & 5.6 & 5.6 & 5.6 & 5.7 & 5.7 & 5.8 & 5.8 & $4.7 \%$ & $<0.001$ \\
\hline Atrial fibrillation (\%) & 6.7 & 7.1 & 7.4 & 7.6 & 7.9 & 8.1 & 8.4 & 8.6 & $27.0 \%$ & $<0.001$ \\
\hline Microvascular disease (\%) & 25.4 & 26.4 & 26.7 & 26.5 & 26.1 & 25.9 & 25.7 & 25.7 & $1.1 \%$ & 0.015 \\
\hline Severe hypoglycaemia (\%) & 2.2 & 2.2 & 2.0 & 1.9 & 1.8 & 1.7 & 1.6 & 1.6 & $-28.9 \%$ & $<0.001$ \\
\hline Kidney disease (\%) & 2.5 & 2.9 & 3.3 & 3.6 & 4.0 & 4.3 & 4.6 & 4.9 & $100.1 \%$ & $<0.001$ \\
\hline Cancer $(\%)$ & 15.4 & 16.0 & 16.3 & 16.8 & 17.3 & 17.8 & 18.2 & 18.7 & $21.4 \%$ & $<0.001$ \\
\hline Lower limb amputations (\%) & 0.4 & 0.4 & 0.4 & 0.5 & 0.5 & 0.5 & 0.5 & 0.5 & $28.5 \%$ & $<0.001$ \\
\hline CVD-preventive drugs ${ }^{\mathrm{a}}(\%)$ & 83.2 & 84.0 & 84.8 & 85.1 & 84.8 & 84.5 & 84.3 & 84.1 & $1.1 \%$ & 0.404 \\
\hline Antihypertensives (\%) & 71.9 & 73.2 & 74.4 & 75.0 & 74.9 & 75.0 & 75.1 & 75.1 & $4.5 \%$ & 0.009 \\
\hline Statins $(\%)$ & 46.4 & 49.7 & 52.9 & 55.2 & 55.8 & 55.9 & 55.9 & 56.0 & $20.8 \%$ & 0.005 \\
\hline Low-dose aspirin (\%) & 42.9 & 43.5 & 43.8 & 43.0 & 41.4 & 39.3 & 37.5 & 35.4 & $-17.6 \%$ & 0.002 \\
\hline
\end{tabular}

Changes are calculated for original values before approximation to one decimal

NSTEMI, non-ST-elevation myocardial infarction; STEMI, ST-elevation myocardial infarction

${ }^{a}$ Any of antihypertensives or statins or low-dose aspirin

the general Swedish population, GLD-treated patients with type 2 diabetes had higher event rates for myocardial infarction, stroke, all-cause death, atrial fibrillation and heart failure: approximately 1.7-, 1.5-, 1.3-, 1.2- and 1.8-fold, respectively (Table 4, Fig. 2). The same pattern was seen in both men and women, although with somewhat higher risk ratios for women than men with type 2 diabetes but with no significant interaction for $\operatorname{sex}$ ( $p$ for interaction $>0.25$; women approximately $1.7-, 1.4-$, 1.3-, 1.2- and 1.7-fold higher and men approximately 1.5-, 1.4-, 1.2-, 1.1- and 1.6-fold higher event rates, respectively; data by sex not shown in tables). From 2006 to 2013 there has been an improvement in life-years lost among women, although no major changes were observed among men (Fig. 3). We observed a slightly improved life expectancy in both the type 2 diabetes and the general population over time (Table 5).

\section{Discussion}

This nationwide observational registry study reports three important findings: a surprisingly high increase in the prevalence of GLD-treated type 2 diabetes; continued high prevalence and risks of diabetes-related disease despite relatively high life expectancy; and important changes in the use of GLDs with increased use of insulin and a decreased use of sulfonyl urea.

The prevalence of GLD-treated type 2 diabetes increased by $61 \%$ (from $2.7 \%$ in 2006 to $4.4 \%$ in 2013 , or by approximately $0.2 \%$ each year). This increase in prevalence was not explained by a parallel increase in incidence, which remained stable during the same time period. In 2013, the prevalence was $4.4 \%$ and the incidence 399 per 100,000 inhabitants. A recent report from Sweden has also reported increasing diabetes prevalence, although at somewhat different rates from those described here. Jansson et al analysed the prevalence of pharmacologically treated diabetes (both type 1 and type 2 combined) during 2005 to 2013 and found on average a $27 \%$ higher prevalence and 10\% higher incidence than our results showed [8]. A possible explanation for this discrepancy is that in contrast to Jansson et al, who used the PDR only, we used information from several nationwide data sources (the PDR, the Cause of Death Register and the National Patient Register) to assess diabetes diagnosis and censor patients with type 2 
Table 3 Use of glucose-lowering treatment and monitoring strips in patients with type 2 diabetes in the study population

\begin{tabular}{llllllllll}
\hline & 2006 & 2007 & 2008 & 2009 & 2010 & 2011 & 2012 & 2013 & $\begin{array}{l}\text { Change (\%) from } \\
2006 \text { to 2013 }\end{array}$ \\
& & & & & & & & \\
Number of patients & 207,303 & 227,837 & 249,495 & 270,284 & 293,013 & 313,599 & 334,360 & 352,436 \\
Metformin (\%) & 68.0 & 68.3 & 69.6 & 69.8 & 70.7 & 70.5 & 70.1 & 69.4 & 2.0 \\
Insulin secretagogues (\%) & 44.4 & 38.9 & 34.2 & 30.1 & 26.8 & 24.1 & 22.0 & 20.4 & -54.0 \\
$\quad$ Sulfonylurea & 38.7 & 33.1 & 28.6 & 25.0 & 22.6 & 20.6 & 18.9 & 17.3 & -55.2 \\
$\quad$ Metiglinides & 6.2 & 6.3 & 6.0 & 5.4 & 4.6 & 3.8 & 3.4 & 3.2 & -47.8 \\
Insulin (\%) & 21.7 & 23.7 & 25.2 & 26.4 & 27.1 & 27.5 & 27.6 & 28.0 & 29.1 \\
$\quad$ Short-acting & 5.4 & 5.8 & 6.3 & 6.6 & 6.8 & 6.8 & 6.9 & 7.1 & 31.2 \\
$\quad$ Medium-to-long-acting & 10.7 & 10.9 & 11.0 & 11.4 & 12.2 & 12.7 & 13.1 & 13.7 & 28.0 \\
$\quad$ Short-to-medium-to-long-acting & 9.3 & 10.2 & 10.7 & 10.9 & 11.0 & 10.9 & 10.6 & 10.2 & 10.1 \\
$\quad$ Long-acting (\%) & 3.2 & 4.2 & 5.2 & 5.7 & 5.5 & 5.4 & 5.4 & 5.5 & 71.1 \\
Incretin-based drugs (\%) & 0.0 & 0.6 & 2.2 & 3.0 & 4.2 & 5.8 & 7.2 & 8.7 & - \\
$\quad$ DPP-4 inhibitor & 0.0 & 0.6 & 2.0 & 2.7 & 3.5 & 4.4 & 5.2 & 6.2 & - \\
$\quad$ GLP1-RA & 0.0 & 0.1 & 0.3 & 0.3 & 0.8 & 1.6 & 2.3 & 2.8 & - \\
Glitazones (\%) & 5.4 & 4.9 & 3.7 & 2.9 & 2.4 & 1.4 & 1.1 & 1.0 & -82.4 \\
Acarbose (\%) & 1.3 & 1.1 & 0.9 & 0.7 & 0.6 & 0.5 & 0.4 & 0.3 & -73.9 \\
SGLT-2 inhibitor (\%) & 0.0 & 0.0 & 0.0 & 0.0 & 0.0 & 0.0 & 0.0 & 0.2 & - \\
Blood glucose test strips (\%) & 48.7 & 45.6 & 45.0 & 42.8 & 39.9 & 37.2 & 34.4 & 32.7 & -32.9 \\
\hline
\end{tabular}

Changes are calculated for original values before approximation to one decimal

DPP-4, dipeptidyl peptidase-4; GLP1-RA, glucagon-like peptide-1 receptor agonist; SGLT-2, sodium-glucose cotransporter 2

diabetes; this probably resulted in more accurate estimates of prevalence and incidence. We excluded most individuals with type 1 diabetes, in contrast to Jansson et al, which not only explains differences in prevalence but also the higher mean age reported by us compared with the former study, 67 vs 64 years, respectively.

There have been several national reports on diabetes prevalence and incidence, with different findings. Some describe an increased prevalence of diabetes [8, 12-17]; the majority report a stable incidence $[12,13,15,16,18]$, some an increasing incidence [19, 20] and one a decreasing incidence [8]. Explanations for these discrepant findings could be related to the decade in which the studies were performed, the definitions applied, the type of register used and the ethnic composition of the population. For instance, Gregg et al showed a significant increase in the incidence of diabetes, both type 1 and type 2, in the US population with the highest lifetime risk of diabetes in the Hispanic population, suggesting that the variation in reported incidences of type 2 diabetes may depend at least in part on ethnicity [4].
Fig. 1 Trends in prescriptions for glucose-lowering treatment for type 2 diabetes filled in Sweden, 2006-2013. DPP-4, dipeptidyl peptidase-4; GLP1-RA, glucagon-like peptide-1 receptor agonist; SGLT-2, sodium-glucose cotransporter 2; SU, sulfonylurea

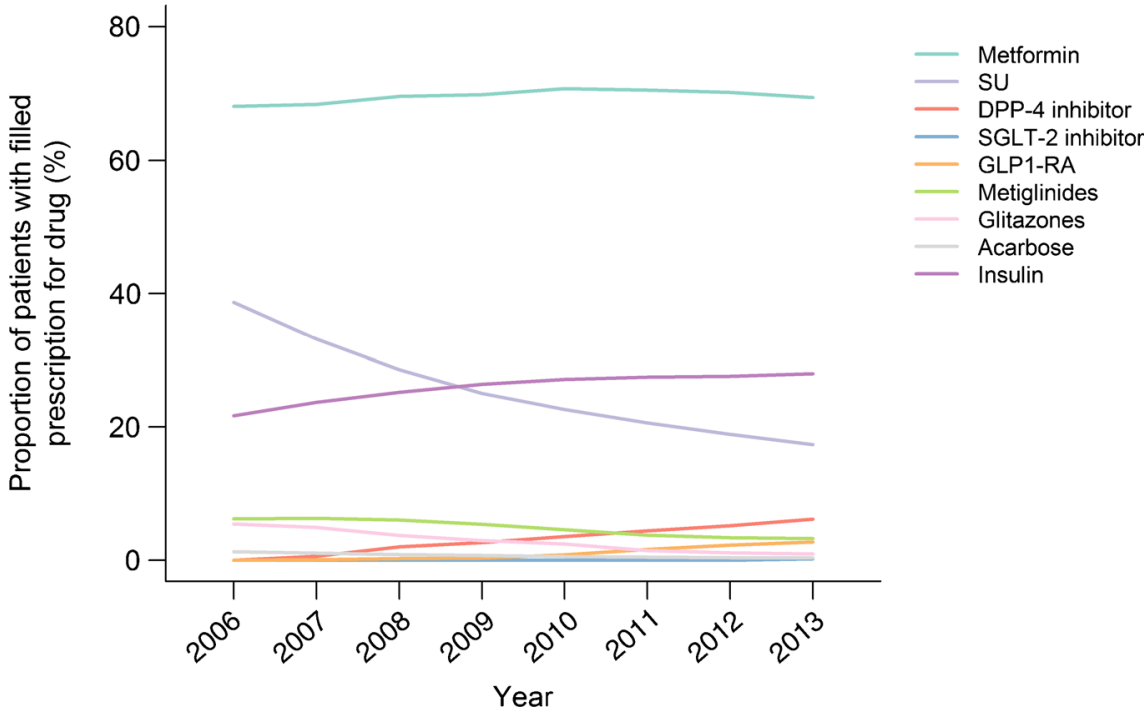




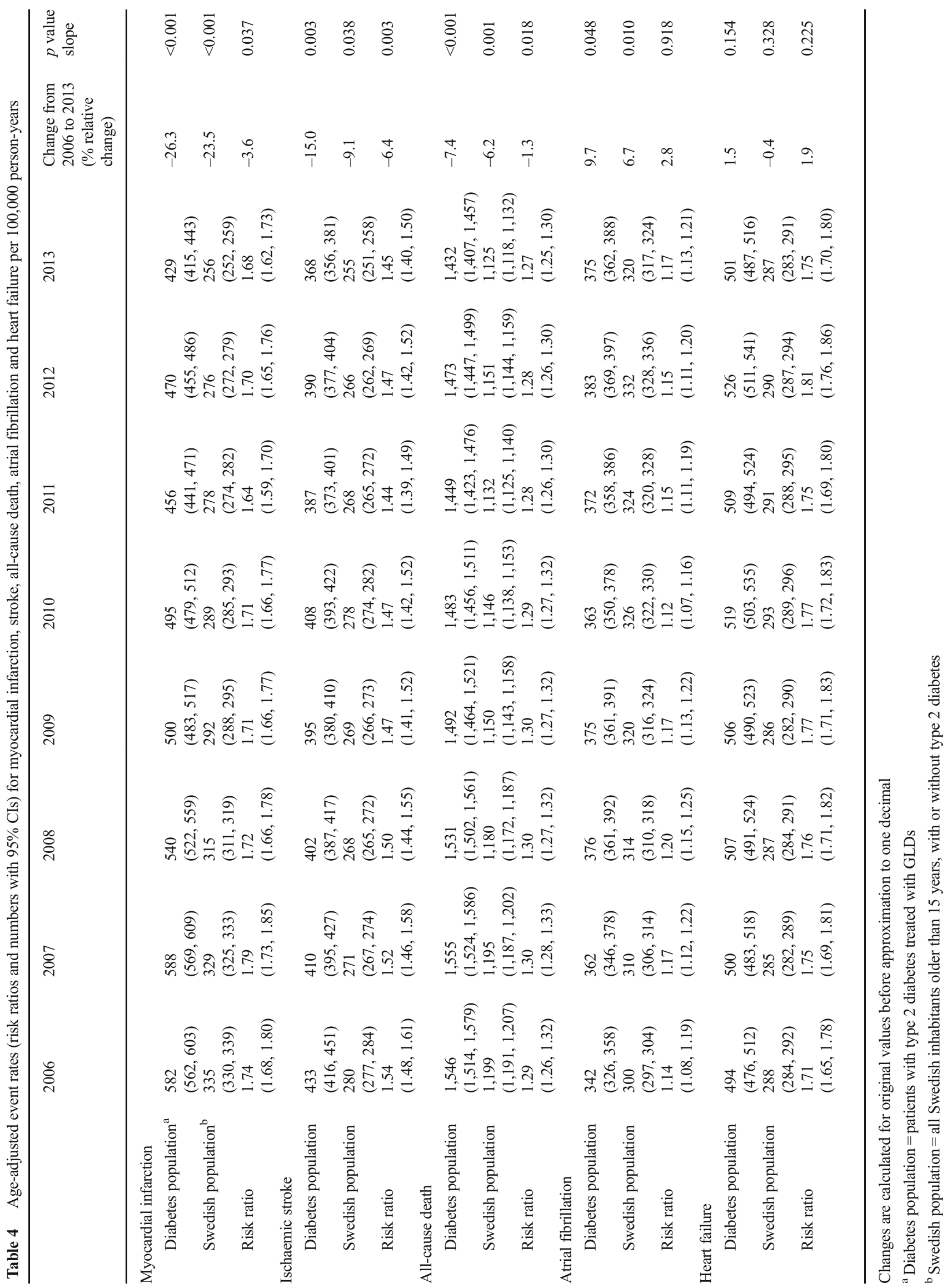


Fig. 2 Age-standardised risk ratio of myocardial infarction, ischaemic stroke, atrial fibrillation, heart failure and allcause death in patients with type 2 diabetes and in the general population in Sweden, 20062013. Shaded areas depict $95 \%$ CIs. $y$-axis is on a logarithmic scale

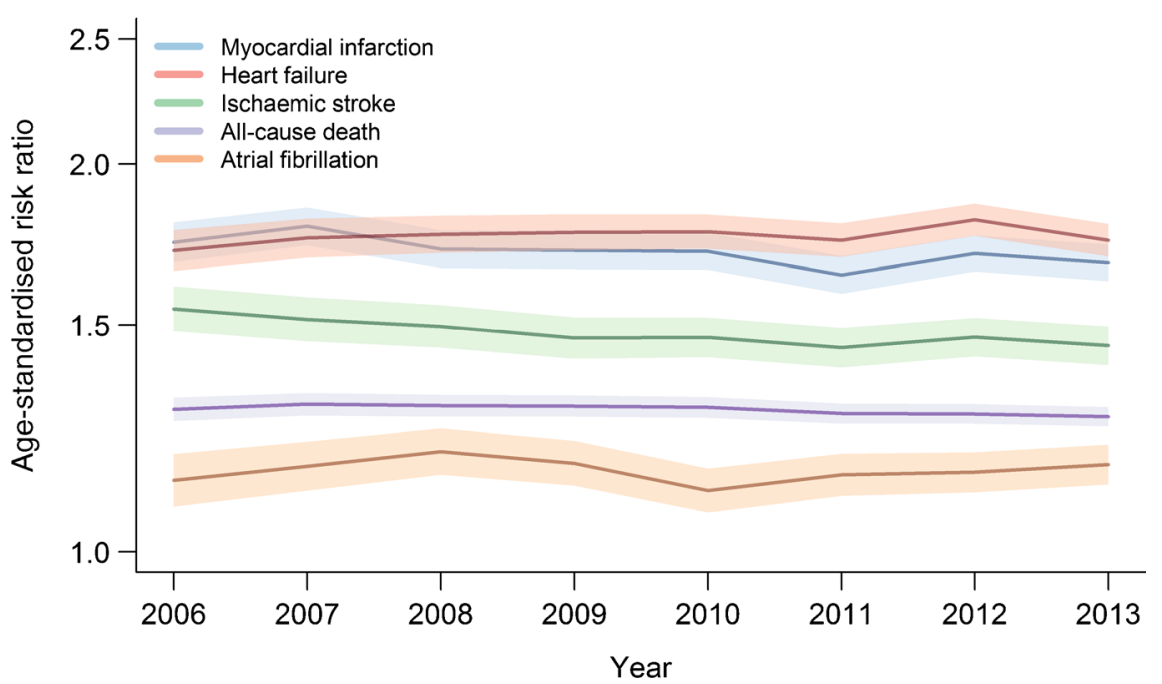

Explanations for the differing prevalence and incidence estimates of type 2 diabetes could also be influenced by demographic changes (such as an ageing population and immigration), effects of changes in treatment patterns (such as earlier GLD initiation [6]) and increased survival rates in the diabetes population. Studies from Canada, Finland, the UK and the USA have reported declining all-cause mortality in the diabetic population but increasing incidence of diabetes [4, 21-23], suggesting that there has been successful treatment of diabetes and diabetes-related complications but insufficient lifestyle-changing programmes to prevent diabetes.

In the present report, the increasing prevalence could partially be explained by reduced mortality during the study period, resulting in a reduced loss of patients, while the incidence remained stable. However, changes in the relatively smaller outflow of patients with type 2 diabetes (mortality approximately 10,000 per year, Table 1) would have a marginal effect

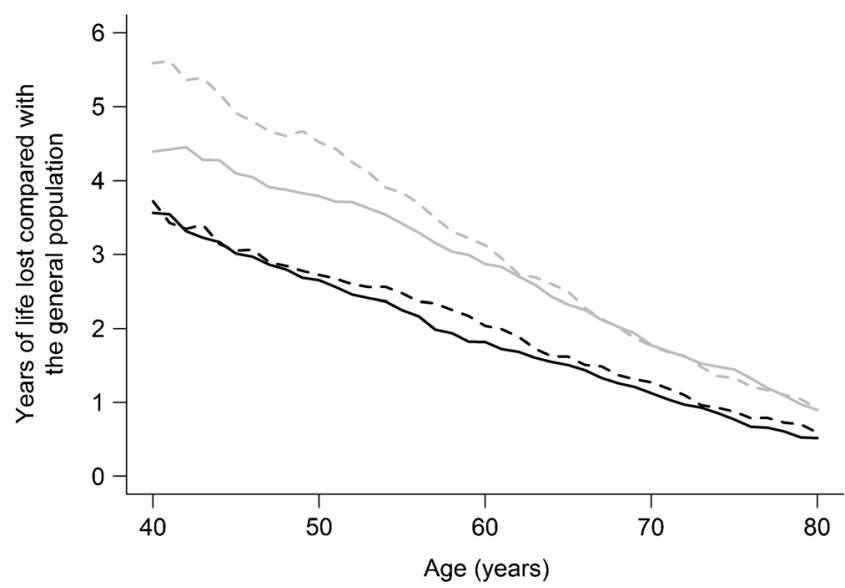

Fig. 3 Years of life lost in men and women with type 2 diabetes compared with the general population in 2006 and 2013. Solid black line, men with type 2 diabetes 2013; dashed black line, men with type 2 diabetes 2006; solid grey line, women with type 2 diabetes 2013; dashed grey line, women with type 2 diabetes 2006 on the total number of patients compared with the much larger patient inflow (incidence of GLD-treated type 2 diabetes, approximately 30,000 per year, Table 1 ). Also, when agestratifying the type 2 diabetes population below and above 70 years, we could not detect any differences in prevalence increase or mortality rates that could add any significant explanation to the increase in prevalence. Earlier GLD treatment could also be an explanation, but when assessing the characteristics of newly treated patients we could not observe a clinically significant change in treatment onset age from 2006 to 2013 (ESM Table 2). Interestingly, the Swedish National Diabetes Registry (NDR) has reported that onset age of type 2 diabetes has not changed in the last years, which in turn would suggest the assumption that time from diagnosis to treatment has not changed much [6]. Diagnostic criteria could also potentially influence the prevalence, however, no guideline change in $\mathrm{HbA}_{1 \mathrm{c}}$ as a diagnostic criterion during the observation time was performed. However, the national guidelines on diabetes treatment was changed in 2010 to recommend the use of metformin as first-line therapy in type 2 diabetes and advocate against use of self-glucose tests in patients with non-insulin treatment, explaining the drop in blood glucose test strips. To the best of our knowledge, this is one of the first reports illustrating increasing prevalence of GLD-treated type 2 diabetes patients while incidence is unchanged and we believe that the main reason for this finding is that the inflow into the prevalent pool far exceeds the mortality outflow from the prevalent pool. Thus, we suggest the occurrence of new cases to be the leading explanation for the steep increase in prevalence.

The second important finding in the present study is the continued high prevalence of CVD and microvascular disease, and high risk of cardiovascular complications and death in the population with type 2 diabetes compared with the general Swedish population, in line with reports from other countries [24].

Annual prevalence of CVD and microvascular disease in the population with type 2 diabetes has been relatively stable 
Table 5 Comparison of life expectancy in 67-year-old patients with and without type 2 diabetes in Sweden

\begin{tabular}{lccccccccc}
\hline & 2006 & 2007 & 2008 & 2009 & 2010 & 2011 & 2012 & 2013 & Change from 2006 to 2013 \\
\hline Life expectancy of diabetes population $^{\mathrm{a}}$, years & 82.8 & 82.8 & 83.0 & 83.3 & 83.2 & 83.4 & 83.4 & 83.7 & +0.9 years \\
Life expectancy of Swedish population $^{\mathrm{b}}$, years & 84.8 & 84.9 & 85.0 & 85.2 & 85.2 & 85.4 & 85.3 & 85.5 & +0.7 years \\
Difference, years & -2.0 & -2.1 & -2.0 & -1.9 & -2.0 & -2.0 & -1.9 & -1.8 & $-10 \%$ \\
\hline
\end{tabular}

${ }^{\mathrm{a}}$ Diabetes population $=$ patients with type 2 diabetes treated with GLDs

${ }^{\mathrm{b}}$ Swedish population $=$ all Swedish inhabitants older than 15 years, with or without type 2 diabetes

over recent years. However, we noted a marked increase in prevalence of atrial fibrillation $(+28 \%)$, kidney disease $(+96 \%)$ and cancer $(+21 \%)$. It is not possible to deduce the explanation for this from the evidence gathered in this registry-based study.

Despite improved diabetes and cardiovascular care in Sweden in recent years [6, 24], and despite our findings of increased life expectancy and favourable trends in mortality and rates of CVD, we observed a still-remaining increased risk of myocardial infarction (1.7-fold), stroke (1.5-fold), heart failure (1.8-fold), atrial fibrillation (1.2-fold) and all-cause death (1.3-fold) in the population with type 2 diabetes compared with the general population. Gregg et al reported ageadjusted risks of myocardial infarction (1.8-fold) and stroke (1.5-fold) in 2010 [25]. Interestingly, despite being conducted in two different countries, these two studies found remarkably similar cardiovascular risks related to diabetes in a modern setting. Life-years lost is still a challenge, especially in patients who have type 2 diabetes at a younger age. We also show that women with diabetes have increased life-years lost compared with men, similar to European Union data [26], but also that an improvement has occurred over time in women, although not in men (Fig. 3). This sex difference in life-years lost due to diabetes could be explained in part by the higher longevity in women without diabetes, but a generally higher cardiovascular risk profile already at diabetes diagnosis seen in younger women compared with men could be another explanation [27]. The positive change in life-years lost in women over time might be explained by a more careful handling of risk factor clusters in women in the latter years due to an increased awareness of the high cardiovascular risk observed in women with diabetes [27].

The improved pharmacological treatment for the prevention of CVD in patients with type 2 diabetes, such as the increased use of statins, might explain some of the favourable trends over time for myocardial infarction, stroke and allcause-death compared with the general population, but more needs to be done in this area. Furthermore, the CVD and microvascular disease burden did not improve during the study period, which is probably a reflection of improved preventive treatments and increased survival rates from acute coronary syndromes even in those with diabetes in the later decades [28]. Together, these observations highlight the need for optimised and new preventive strategies to further reduce the difference in mortality and $\mathrm{CV}$ risk between patients with and without diabetes.

The third important finding in this study is the change in pattern of GLD treatment in recent years, with an increase of almost $30 \%$ in the use of insulin and a $55 \%$ decline in the use of insulin sulfonylurea. Explanations for the increase in insulin use could include the national medical authorities' objectives of achieving improved blood glucose levels, together with annual open reporting of what has been achieved, resulting in earlier treatment with insulin to meet these goals. Although there was a steep increase in the later study years, the use of incretin-based treatment remained low in 2013 $(9 \%)$. Metformin was, as expected, the GLD with the highest level of filled prescriptions and fairly constantly prescribed. Compared with other European countries, Sweden had the highest use of insulin among GLDs (about 28\%), followed by $\sim 20 \%$ use in the Netherlands, Spain and the UK [29]. The reported decreased use of insulin secretagogues is in line with rest of Europe [29]. Despite the steep increase in insulin use, the use of blood glucose test strips decreased by about $30 \%$, which was an unexpected finding in our study. This raises the question of whether this reflects an unfavourable trend in patients' individual glucose control, which would need to be evaluated in future studies.

Strengths and limitations There are several limitations with registry reports such as this. One is the lack of data on important diabetes variables, such as glycated haemoglobin and duration of diabetes. Furthermore, since there are no available codes for diabetes managed by diet, we lack information on this group of patients, who were not included in the present study. Thus, our defined population of GLD-treated patients with type 2 diabetes is likely to be an underestimation of the true prevalence of type 2 diabetes in Sweden. The type 2 diabetes-free population was considered as the total population without any glucose-lowering prescriptions as defined above. Thus, there could be a misclassification, including diet-treated individuals in the non-diabetes population. However, when taking the large relative differences in group sizes into account, this might have underestimated the true diabetes prevalence and incidence but to a very small degree. 
Furthermore, some individuals classified as having type 1 diabetes in the present study could have been misclassified, further underestimating the true prevalence of type 2 diabetes. Microvascular disease might be treated, to an unknown extent, outside hospital care (e.g. in primary care or private eye clinics) and could therefore be incompletely captured in this study. Cardiovascular risk might be somewhat overestimated in the present analyses since the healthier diabetes group with only diet treatment is not included in the present cohort. However, since the GLD-treated group accounts for $\sim 80 \%$ of the entire type 2 diabetes population, the information on increased cardiovascular risk is highly valuable and identifies the majority and the most vulnerable part of the type 2 diabetes population at risk for CVD [6].

The major strength is the nationwide reach of the study, including $100 \%$ register coverage for cause of hospitalisations, filled prescriptions of drugs and cause of death in Sweden. For example, this is illustrated by the discrepant report on diabetes incidence from the Swedish NDR [6], which reported less than half the incidence found in the present study on pharmacologically treated type 2 diabetes patients. The reason for this might be that the NDR relies on optional registration from primary and secondary healthcare, while our data are based on obligatory registered codes in other national registers with a more accurate estimation.

Conclusions In this nationwide study, a $61 \%$ increase was found in the prevalence of type 2 diabetes treated with GLDs during 2006-2013, while the incidence remained stable. The increase in prevalence is little explained by change in diabetes diagnosis criteria, earlier onset of GLD treatment or change in prolonged longevity, and mainly by the actual occurrence of new type 2 diabetes cases. The use of sulfonylurea to treat diabetes declined while the use of insulin increased, being the second most common drug after metformin. Although there are favourable trends in CVD and slightly prolonged life expectancy, the high prevalence of diabetes-related comorbidities, lifeyears lost and continued higher CVD risk in patients with type 2 diabetes than in the general population highlights the need for optimised and new preventive strategies.

Acknowledgements We are grateful to U. Olsson at Statisticon AB (Uppsala, Sweden), for support with data merging and management of the database, and to S. Jerström and H. Goike at AstraZeneca for logistic support and valuable comments on the manuscript. Editorial support funded by AstraZeneca was provided by Oxford PharmaGenesis.

Contribution statement All authors were involved in the design of the study, the literature research and the data interpretation. AN, JB and DN wrote the first draft of the manuscript. MT performed the data collection and statistical analysis. All authors made critical revision of the manuscript for key intellectual content. All authors gave final approval of the submitted manuscript. The corresponding author (AN) had full access to the data and takes responsibility for the decision to submit for publication.
Funding This study was sponsored by AstraZeneca.

Duality of interest $\mathrm{AN}$ has received research grants from the Swedish Heart-Lung Foundation, the Swedish Diabetes Foundation, honoraria from expert group participation (MSD, Astra Zeneca, Novo Nordisk), none of the aforementioned directly related to this publication. JB holds a full time position as an epidemiologist at AstraZeneca. TN is on the national board for Sanofi, Eli Lilly and NovoNordisk. MT is employed by an independent statistical consultant company, Statisticon AB, Uppsala, Sweden, for which AstraZeneca Nordic-Baltic is a client. JWE was previously employed at AstraZeneca R\&D, Mölndal, Sweden. DN has received consultancy fees from Novo Nordisk, Astra Zeneca and Eli Lilly.

\section{References}

1. IDF (2014) IDF diabetes atlas. Available from www.diabetesatlas. org, accessed 12 December 2015

2. WHO (2014) Global status report on noncommunicable diseases. Available from apps.who.int/iris/bitstream/10665/148114/1/ 9789241564854 eng.pdf?ua $=1$, accessed 5 December 2015

3. Grover SA, Kaouache M, Rempel P et al (2015) Years of life lost and healthy life-years lost from diabetes and cardiovascular disease in overweight and obese people: a modelling study. Lancet Diabetes Endocrinol 3:114-122

4. Gregg EW, Zhuo X, Cheng YJ, Albright AL, Narayan KM, Thompson TJ (2014) Trends in lifetime risk and years of life lost due to diabetes in the USA, 1985-2011: a modelling study. Lancet Diabetes Endocrinol 2:867-874

5. Public Health Agency of Sweden (2014) Folkhälsan i Sverige. Available from www.folkhalsomyndigheten.se/pagefiles/17825/ Folkhalsan-i-Sverige-arsrapport-2014.pdf [article in Swedish], accessed 1 November 2015

6. Gudbjörnsdottir S, Eliasson B, Cederholm J, Zethelius B, Svensson AM, Samuelsson P (2014) Swedish National Diabetes Register annual report 2013. Centre of Registers, Västra Götaland

7. Jansson SP, Andersson DK, Svardsudd K (2007) Prevalence and incidence rate of diabetes mellitus in a Swedish community during 30 years of follow-up. Diabetologia 50:703-710

8. Jansson SP, Fall K, Brus O et al (2015) Prevalence and incidence of diabetes mellitus: a nationwide population-based pharmaco-epidemiological study in Sweden. Diabet Med 32:1319-1328

9. Wandell PE, Carlsson A, Steiner KH (2010) Prevalence of diabetes among immigrants in the Nordic countries. Curr Diabetes Rev 6: 126-133

10. Chiang LC (1984) The life table and its applications. Robert E. Krieger Publishing Company, Malabar

11. Team RDC (2011) R: a language and environment for statistical computing. R Foundation for Statistical Computing, Vienna

12. Berger B, Stenstrom G, Sundkvist G (1999) Incidence, prevalence, and mortality of diabetes in a large population. A report from the Skaraborg Diabetes Registry. Diabetes Care 22:773-778

13. Monesi L, Baviera M, Marzona I et al (2012) Prevalence, incidence and mortality of diagnosed diabetes: evidence from an Italian population-based study. Diabet Med 29:385-392

14. Selvin E, Parrinello CM, Sacks DB, Coresh J (2014) Trends in prevalence and control of diabetes in the United States, 19881994 and 1999-2010. Ann Intern Med 160:517-525 
15. Strom H, Selmer R, Birkeland KI et al (2014) No increase in new users of blood glucose-lowering drugs in Norway 2006-2011: a nationwide prescription database study. BMC Public Health 14:520

16. Thunander M, Petersson C, Jonzon K et al (2008) Incidence of type 1 and type 2 diabetes in adults and children in Kronoberg, Sweden. Diabetes Res Clin Pract 82:247-255

17. Xu Y, Wang L, He J et al (2013) Prevalence and control of diabetes in Chinese adults. JAMA 310:948-959

18. CDC (2012) Diabetes national data US. Available from www.cdc. gov/diabetes/statistics/incidence national.htm, accessed 17 October 2015

19. Gonzalez EL, Johansson S, Wallander MA, Rodriguez LA (2009) Trends in the prevalence and incidence of diabetes in the UK: 19962005. J Epidemiol Community Health 63:332-336

20. Ryan R, Newnham A, Khunti K, Majeed A (2005) New cases of diabetes mellitus in England and Wales, 1994-1998: database study. Public Health 119:892-899

21. Evans JM, Barnett KN, Ogston SA, Morris AD (2007) Increasing prevalence of type 2 diabetes in a Scottish population: effect of increasing incidence or decreasing mortality? Diabetologia 50:729-732

22. Lind M, Garcia-Rodriguez LA, Booth GL et al (2013) Mortality trends in patients with and without diabetes in Ontario, Canada and the UK from 1996 to 2009: a population-based study. Diabetologia $56: 2601-2608$
23. Lipscombe LL, Hux JE (2007) Trends in diabetes prevalence, incidence, and mortality in Ontario, Canada 1995-2005: a populationbased study. Lancet 369:750-756

24. Chung SC, Gedeborg R, Nicholas O et al (2014) Acute myocardial infarction: a comparison of short-term survival in national outcome registries in Sweden and the UK. Lancet 383:1305-1312

25. Gregg EW, Li Y, Wang J et al (2014) Changes in diabetes-related complications in the United States, 1990-2010. N Engl J Med 370: $1514-1523$

26. Emerging Risk Factors Collaboration, Seshasai SR, Kaptoge $\mathrm{S}$ et al (2011) Diabetes mellitus, fasting glucose, and risk of cause-specific death. N Engl J Med 364:829-841

27. Peters SA, Huxley RR, Sattar N, Woodward M (2015) Sex differences in the excess risk of cardiovascular diseases associated with type 2 diabetes: potential explanations and clinical implications. Curr Cardiovasc Risk Rep 9:36

28. Norhammar A, Lindback J, Ryden L et al (2007) Improved but still high short- and long-term mortality rates after myocardial infarction in patients with diabetes mellitus: a time-trend report from the Swedish Register of Information and Knowledge about Swedish Heart Intensive Care Admission. Heart 93: $1577-1583$

29. Heintjes E, Overbeek J, Blin P et al (2014) Type 2 diabetes treatment patterns across Europe. Value Health 17:A359 